\title{
The influence of nano-particle tracers on the slip length measurements by microPTV
}

\author{
Xu Zheng · Gao-Pan Kong · Zhan-Hua Silber-Li
}

Received: 2 July 2012 / Revised: 18 December 2012 / Accepted: 12 March 2013

(C)The Chinese Society of Theoretical and Applied Mechanics and Springer-Verlag Berlin Heidelberg 2013

\begin{abstract}
Direct measurement of slip length is based on the measured fluid velocity near solid boundary. However, previous micro particle image velocimetry/particle tracking velocimetry (microPIV/PTV) measurements have reported surprisingly large measured near-wall velocities of pressuredriven flow in apparent contradiction with the no-slip hypothesis and experimental results from other techniques. To better interpret the measured results of the microPIV/PTV, we performed velocity profile measurements near a hydrophilic wall $(z=0.25-1.5 \mu \mathrm{m})$ with two sizes of tracer particles $(\phi 50 \mathrm{~nm}$ and $\phi 200 \mathrm{~nm})$. The experimental results indicate that, at less than $1 \mu \mathrm{m}$ from the wall, the deviations between the measured velocities and no-slip theoretical values obviously decrease from $93 \%$ of $\phi 200 \mathrm{~nm}$ particles to $48 \%$ of $\phi 50 \mathrm{~nm}$ particles. The Boltzmann-like exponential measured particle concentrations near wall were found. Based on the non linear Boltzmann distribution of particle concentration and the effective focus plane thickness, we illustrated the reason of the apparent velocity increase near wall and proposed a method to correct the measured velocity profile. By this method, the deviations between the corrected measured velocities and the no-slip theoretical velocity decrease from $45.8 \%$ to $10 \%$, and the measured slip length on hydrophilic glass is revised from $75 \mathrm{~nm}$ to $16 \mathrm{~nm}$. These results indicated that the particle size and the biased particle concentration distribution can significantly affect near wall velocity measurement via microPIV/PTV, and result in larger measured velocity and slip length close to wall.
\end{abstract}

The project was supported by the National Natural Science Foundation of China (10872203), the National Basic Research Program (2007AC744701) and the CAS Research and Development Program of China (KSCX2-YW-H18).

\section{Zheng · G.-P. Kong · Z.-H. Silber-Li(凷)}

State Key Laboratory of Nonlinear Mechanics,

Institute of Mechanics, CAS, 100190 Beijing, China

e-mail: lili@imech.ac.cn
Keywords MicroPIV/PTV · Slip length · Nano-particle tracer - Particle concentration distribution - Boltzmann distribution

\section{Introduction}

Slip boundary condition has been debated for two hundred years. In microflows, surface effect is dominant, and the slip at a liquid-solid interface becomes an important phenomenon. Based on the common-used Navier slip model, the surface slip velocity $u_{\text {slip }}$ is expressed as [1]

$u_{\text {slip }}=b \times \dot{\gamma}_{\mathrm{w}}=\left.b \frac{\partial u}{\partial z}\right|_{\text {wall }}$,

where $\dot{\gamma}_{\mathrm{w}}$ is the shear rate at wall, $b$ is the slip length, which is defined as the distance to wall where velocity is extrapolated to zero (Fig. 1a). Thus, in the region very close to wall where velocity profile is approximately linear, $b$ can be calculated by velocity profile fitting using Eq. (1). From the model, the measurement of $u_{\text {slip }}$ is then equivalent to the measurement of $b$.

In a series of recent works, micro particle image velocimetry (microPIV) [2,3] and micro particle tracking velocimetry (microPTV) have been used to measure velocities close to solid boundaries. However, these measured slip velocities or slip lengths were rather scattered. For example, Tretheway and Meinhart [4] used a high numerical aperture microPIV system $(N A=1.4)$ with $\phi 300 \mathrm{~nm}$ fluorescent tracers to measure the velocities close to hydrophilic and hydrophobic walls. The closest measured position was $450 \mathrm{~nm}$ away from wall, and approximately $1 \mu \mathrm{m}$ slip length was obtained on a wall with $90^{\circ}$ contact angle (Fig. 1b). Joseph and Tabeling [5] used a high-resolution piezo transducer positioning system (resolution $\Delta z=10 \mathrm{~nm}$ ) in combination with a high numerical aperture objective $(N A=1.3)$ to measure the slip velocity directly at a hydrophilic wall in a microchannel using $\phi 200 \mathrm{~nm}$ particles. The velocity profile in 
the vertical direction was measured down to $z=300 \mathrm{~nm}(z$ is the distance from the wall). But they found that the measured velocities for $z<1 \mu \mathrm{m}$ were noticeably larger than the expected theoretical no-slip values and reported a slip length of $50 \pm 50 \mathrm{~nm}$ for a hydrophilic glass wall (Fig. 1b). Zheng and Silber-Li [6] also used a high resolution piezo transducer system with a high numerical aperture objective $(N A=1.35)$ to measure near-wall velocity profiles in 14 horizontal planes in a rectangular microchannel with hydrophilic walls using $\$ 200 \mathrm{~nm}$ particles, and compared them with the theoretical no-slip predictions. The measured velocities in the main flow region were in good agreement with the theoretical no-slip velocity profile, however, when $z<1 \mu \mathrm{m}$ the experimental data were approximately $18 \%$ larger than those predicted by the theoretical no-slip velocity profile. A hydrophilic wall is considered to well approximate a no-slip boundary condition for flows of pure water and slip lengths of less than $5 \mathrm{~nm}$ are expected, which is also confirmed by molecular dynamic simulation [7,8]. Surface force apparatus (SFA) or atomic force microscope (AFM) was also used to measure the slip length as an alternative method, and these methods reported only approximately 1$10 \mathrm{~nm}$ slip length on hydrophilic surface, which is consistent with the theoretical prediction $[9,10]$. Figure $1 \mathrm{~b}$ summarizes the results and shows clearly the large deviation between the measured data via microPIV/PTV and the results obtained by SFA/AFM. Thus microPIV measurements reporting slip lengths $O(\sim 100 \mathrm{~nm})$ may appear to be in considerable problem.
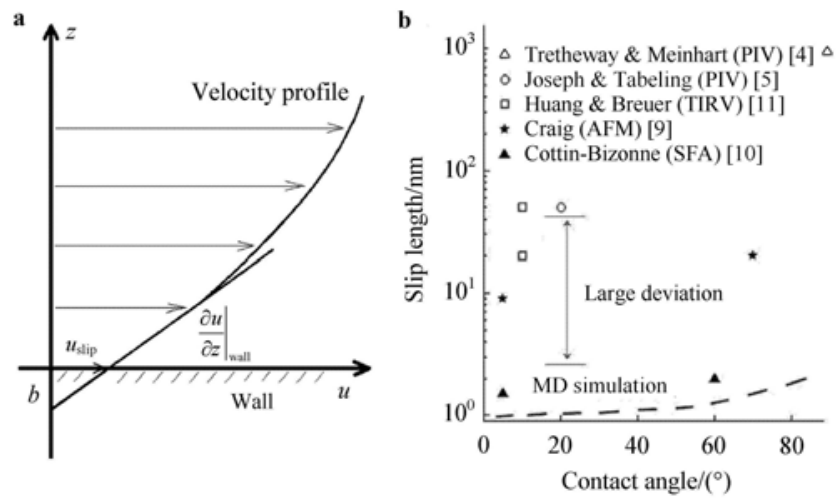

Fig. 1 a A schematic diagram of Navier's slip model and b a summary of results of measured slip length on surfaces with contact angle from $0^{\circ}-90^{\circ}$ by microPIV/PTV and by SFA/AFM, where the result from molecular simulation (MD, dash line) is also shown. The large deviation between the measured slip length by microPIV and by SFA/AFM can be seen clearly

In microPIV/PTV near wall measurements, whether seeding particles can be effectively used to detect local fluid velocity is a crucial problem. Obviously the size of the seeding particle is important. Then, the influence of depth of measurement due to volume illumination on fluorescent particles was considered by Olsen and Adrian [12]. Because the measured velocities are averaged from all particle tracked within the depth of measurement, the accuracy of the measured velocity is related to the velocity field uniformity. In their experiments, a $20 x / N A=0.5$ objective was used, and the depth of measurement was approximately $10 \mu \mathrm{m}$. Even the spatial resolution can be improved by using a $100 x / N A=1.35$ [6] objective, giving a depth of measurement of the order of $1 \mu \mathrm{m}$, which is still comparable with the characteristic dimension of the near wall region. Many other physical effects, such as electrostatic effects [13], particle depletion layer $[5,14]$ and the hydrodynamic effect of the particles $[11,15]$ have been studied. Furthermore, Zheng and Silber-Li [16] studied the behavior of particle tracer concentration distribution in a shear flow. But up to now, no results have been reported to show the influence of particle concentration on near wall velocity measurement. Therefore it is necessary to clarify the influences of particle sizes, the particle concentration distribution, the depth of measurement and the shear flow on microPIV/PTV measurement near wall and improve its measured velocity interpretation in measurements close to a solid boundary.

In this paper, we focus on the aforementioned influences on the near wall velocities measured by microPIV/PTV. To explore the influence of tracer particles size, two diameters of particles $d_{\mathrm{p}}=50 \mathrm{~nm}$ and $d_{\mathrm{p}}=200 \mathrm{~nm}$ are used in pure water driven by pressures. The velocity measurement positions are close to the wall region $(0.25 \mu \mathrm{m} \leqslant z \leqslant$ $1.5 \mu \mathrm{m})$. We also measure the particle concentration distribution, which is obviously non-uniform and similar to Boltzmann distribution along $z$ near wall. In order to limit the influence of depth of measurement, we propose to use the effective focus plane thickness which limited the observation thickness to $0.4 \mu \mathrm{m}$. Based on the observed exponential particle concentration distribution, effective focus plane thickness and the shear flow field, we explain the deviation between the measured data and the no-slip theoretical values, and propose a velocity profile correction method. In Sect. 2, the experimental apparatus and method are elucidated. The experimental results, an explanation of the deviations of measured velocity, a measured velocity profile correction and the measured slip length are presented in Sect. 3. The conclusions are summarized in Sect. 4.

\section{Experimental apparatus and method}

\subsection{Experimental apparatus}

The measurements were carried out using a microflow measurement system at the LNM, Institute of Mechanics, Chinese Academy of Sciences $[6,16]$. Taking account of the low velocity near wall, we used a particle tracking velocimetry (PTV) technique. The measurement system includes a fluorescent inverted microscope (Olympus IX71), an EMCCD (Andor DV885) and a piezo-transducer (LVPZT E665). A pressure driven flow approach is used. The precision of 
the pressure measurement is $0.3 \%$, and the applied pressure ranges from $0.8 \mathrm{kPa}$ to $2.4 \mathrm{kPa}$.

The rectangular PDMS-glass hybrid microchannels $(19.1 \pm 0.1) \mu \mathrm{m}$ in height, $(56.0 \pm 0.2) \mu \mathrm{m}$ in width and $(28.00 \pm 0.02) \mathrm{mm}$ in length) are used. The contact angle (CA) on the glass substrate is $20^{\circ}-30^{\circ}$. Streamwise and spanwise directions are set as the $x$ and $y$ axes of the microchannel coordinate system, respectively, and vertical direction is $z$. The original point is located at the center point of the width at the bottom of the microchannel (Fig. 2).

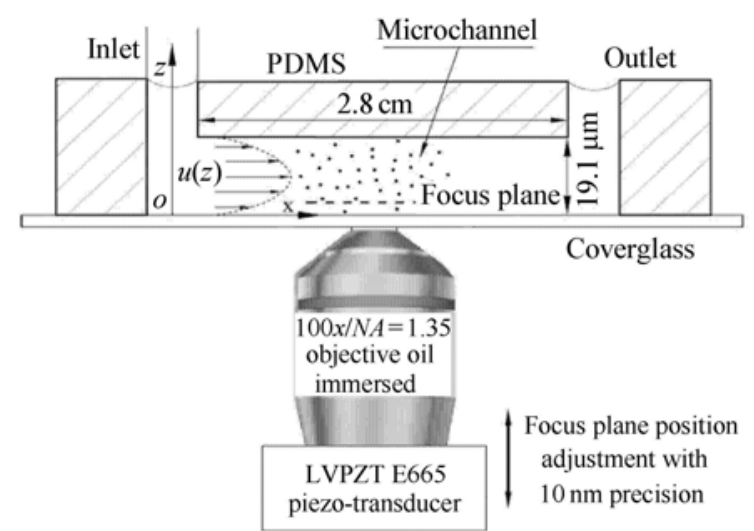

Fig. 2 Schematic diagram of the experimental setup. The measured position is $0.25-1.5 \mu \mathrm{m}$ to a glass substrate in a microchannel ( $56.0 \mu \mathrm{m} \times 19.1 \mu \mathrm{m}$, and $2.8 \mathrm{~cm}$ in length). A piezo-transducer was mounted under the objective to control the position of focus plane with a precision of $10 \mathrm{~nm}$

Fluorescent polystyrene particles (Duke Scientific Corporation) of $\phi 50 \mathrm{~nm}$ and $\phi 200 \mathrm{~nm}$ were used as tracers in the experiments. The exciting and emitted waves are $530 \mathrm{~nm}$ and 600-610 nm, respectively. The dimensionless volume concentrations of the fluorescent particles were approximately $3 \times 10^{-5}-5 \times 10^{-5}$. The particle number densities per unit volume are $1 \times 10^{7} \mu \mathrm{L}^{-1}(\phi 200 \mathrm{~nm})$ and $6 \times 10^{8} \mu \mathrm{L}^{-1}(\phi 50 \mathrm{~nm})$, respectively. Therefore, the density and viscosity of the test liquid are nearly identical to the purified water.

\subsection{Experimental procedure}

\subsubsection{Measurement procedure}

In the experiment, the liquid flow in the microchannel was driven by nitrogen gas. The driving pressure in the range of $1-3 \mathrm{kPa}$ was monitored by a pressure transducer, whose accuracy was $0.3 \%$. The environmental temperature was from $24^{\circ} \mathrm{C}-26^{\circ} \mathrm{C}$ during the experiment, and the temperature measurement was accurate to $\pm 0.1^{\circ} \mathrm{C}$. The experiment was started when the flow was steady. The distance from the measurement position to the inlet of the channel was larger than $1 \mathrm{~cm}$, which was approximately $300 d_{\mathrm{h}}\left(d_{\mathrm{h}}\right.$ is the hydrodynamic diameter of the channel). Therefore, the influence of the inlet was considered negligible and the flow was considered to be fully developed at the measurement location.

\subsubsection{Position of wall}

The velocities were measured near the glass wall at five vertical positions: $z=0.25 \mu \mathrm{m}, 0.5 \mu \mathrm{m}, 0.75 \mu \mathrm{m}, 1.0 \mu \mathrm{m}$, and $1.5 \mu \mathrm{m}$. The method of determination of the wall position, originally proposed by Joseph and Tabeling [5], is the same as that used in our previous experiments $[6,16]$. It is based on the diffraction relation (Lorentzian distribution, Fig. 3) between the intensity of the particle and the distance from the particle to the focus plane. The wall position $(z=0$, showed by the dashed line), also the lowest point of the particle attached to wall, is found by subtracting one particle radius from the maximum intensity position corresponding to the particle center. If the uncertainty of the gray value in an image is \pm 1 (arb. unit in Fig. 3, $y$-axis), then we estimate that the uncertainty of the vertical position determined in the present experiment is approximately $25-40 \mathrm{~nm}$.

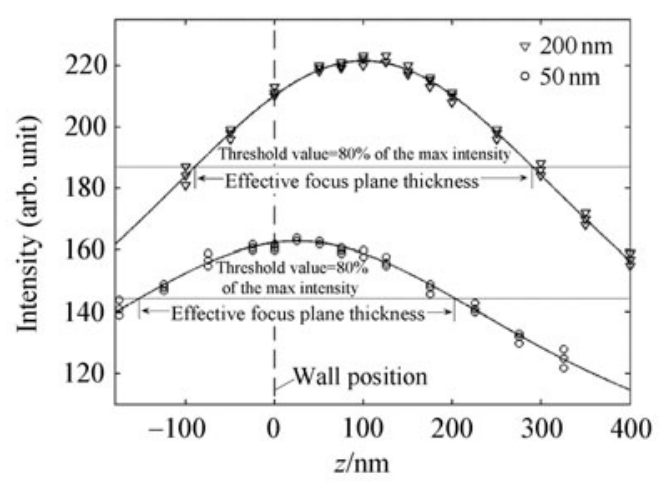

Fig. 3 The intensity distribution of particles adsorbed on wall.The effective focus plane thickness can be determined from the width of the fitting curve upon the threshold line

\subsubsection{Image processing}

In this work we employ a PTV method by recording images of particle streaks in steady flow. The exposure time $\Delta t$ of the CCD was varied from $5 \mathrm{~ms}$ to $25 \mathrm{~ms}$, depending on the vertical position and the flow speed. The particle concentration was very low, so at least 200 images were recorded at each measurement position.

There are two sources of error in the image processing procedures. One appears in determining the position of the two ends of the streak. Although the end positions are dependent on the sharp change of the streak grey-scale value, the tracked position of each end locates randomly around the true position of the particle. Another possible error is due to the Brownian motion of the nano particles, especially for the $\phi 50 \mathrm{~nm}$ particles. However, by ensemble averaging over 200-250 images, these two random errors can be reduced to approximately $1 \%$ of the measured velocities and will thus be neglected. 


\subsection{Effective focus plane thickness}

The common used depth of field [3, 17] defines the thickness of the focus plane that an image can be obtained clearly. But owing to the volume illumination, the fluorescent particles can be caught in a layer thicker than the depth of field in microPIV/PTV. Thus depth of measurement $\delta z_{\mathrm{m}}$ was proposed by Meinhart et al. [18] as $\delta z_{\mathrm{m}}=\frac{3 n_{\mathrm{r}} \lambda}{N A^{2}}+\frac{2 \cdot 16 d_{\mathrm{p}}}{\tan \theta}+d_{\mathrm{p}}(\theta$ is the light collection angle). In the present experiment, for the $d_{\mathrm{p}}=200 \mathrm{~nm}$ particles, $\delta z_{\mathrm{m}} \approx 1.8 \mu \mathrm{m}$; for the $d_{\mathrm{p}}=50 \mathrm{~nm}$ particles, $\delta z_{\mathrm{m}} \approx 1.6 \mu \mathrm{m}$.

However, 1.6-1.8 $\mu \mathrm{m}$ depth of measurement is too thick to use in near wall velocity measurement (with $1 \mu \mathrm{m}$ to wall). We propose the effective focus plane thickness $\Delta z$ instead of the depth of measurement. Based on the measured particle intensity distribution, a threshold of the grey-scale value was chosen to filter out the out-of-plane particles. This threshold value was set as $80 \%$ of the maximum grey-scale value in the image series at each position. To estimate the thickness limited by the threshold, we use the intensity distribution of the particles attached to wall (Fig. 3). According to the width of the Lorentzian fitting curve upon the threshold line, the effective focus plane thickness $(\Delta z)$ can be limited to approximately $400 \mathrm{~nm}$. Thus, the method using effective focus plane thickness can decrease the influence of the outof-focus effect in the measurement.

\section{Experimental results}

3.1. Near wall measurement of velocities using $\phi 200 \mathrm{~nm}$ and $\phi 50 \mathrm{~nm}$ particles

The present measurements span the region from $z^{+}=7.5$ to $z^{+}=1.25\left(z^{+}=z / 2 r, r\right.$ is the radius of the particle $)$ using $\phi 200 \mathrm{~nm}$ particles. The horizontal velocity measurements (in the $x-y$ planes) were carried out at five positions $\left(z^{+}=1.25,2.5,3.75,5.0\right.$, and 7.5), and under three different driven pressures $(\Delta p=0.8 \mathrm{kPa}, 1.6 \mathrm{kPa}$, and $2.4 \mathrm{kPa})$. The Reynolds numbers $R e=0.04-0.13$ based on the hydrodynamic diameter of the channel. For $\phi 50 \mathrm{~nm}$ particles, the measurement conditions were the same as above, but the non-dimensional distances from the wall were at $z^{+}=5,10$, 15, 20, and 30 .

The experimental velocity data at the $x-y$ planes are shown in Fig. 4 for three positions (a, b, c for $\phi 200 \mathrm{~nm}$ particles, and $\mathrm{d}, \mathrm{e}, \mathrm{f}$ for $\phi 50 \mathrm{~nm}$ particles) and compared with the 3D theoretical no-slip velocity profiles [19]

$$
\begin{aligned}
u_{x}(y, z)= & \frac{4 h^{2} \Delta p}{\pi^{3} \mu L} \sum_{n=1,3,5, \ldots}^{\infty} \frac{1}{n^{3}}\left(1-\frac{\cosh \left(n \pi \frac{y}{h}\right)}{\cosh \left(n \pi \frac{w}{2 h}\right)}\right) \\
& \times \sin \left(n \pi \frac{z}{h}\right),
\end{aligned}
$$

where $h$ is the height of the channel, and $L$ is the length of the channel. In Figs. $4 \mathrm{a}$ to $4 \mathrm{c}$, we notice that, as $z$ decreases, the deviation between measured data and theoretical values increases. To illustrate the discrepancies in a velocity profile, the average relative deviation is defined as $\bar{\varepsilon}=\frac{1}{n} \sum_{n=1}^{n} \frac{\left(u_{\mathrm{exp}, n}-u_{\mathrm{theo}, n}\right)}{u_{\mathrm{theo}, n}}, n$ is the number of data in a profile. The values of $\bar{\varepsilon}$ in different horizontal planes are given in Table 1. For $\phi 200 \mathrm{~nm}$ particles, at the highest distance from the wall $z^{+}=7.5, \bar{\varepsilon} \approx 1.7 \%$. However, for the closest location $z^{+}=1.25, \bar{\varepsilon} \approx 93.1 \%$, and we clearly see the values of the measured velocities at $z^{+} \leqslant 5$ are larger than the theoretical values for a no-slip boundary condition.

The measurement for $\phi 50 \mathrm{~nm}$ particles showed smaller deviations between measured data and theoretical values compared with the measurement for $\phi 200 \mathrm{~nm}$ particles (Figs. $4 \mathrm{~d}-4 \mathrm{e})$. Table 1 also shows the quantities of the average relative deviations $\bar{\varepsilon}$ at each horizontal plane. $\bar{\varepsilon}=-0.7 \%$ at $z^{+}=30$, but at $z^{+}=5, \bar{\varepsilon}=45.8 \%$. It is clear that while measurements for $z^{+}>10$ agree well with theory, the values of the measured velocities at $z^{+}<10$ are significantly larger than the theoretical values for a no-slip boundary condition.

For three driven pressures, the same tendencies are obtained. So only experimental results under $\Delta p=2.4 \mathrm{kPa}$ are shown above.

Table 1 The average relative deviation $\bar{\varepsilon}$ at each horizontal plane

\begin{tabular}{lllllll}
\hline \multirow{2}{*}{$\phi 200 \mathrm{~nm}$} & Position & $z^{+}=1.25$ & $z^{+}=2.5$ & $z^{+}=3.75$ & $z^{+}=5.0$ & $z^{+}=7.5$ \\
\cline { 2 - 7 } & $\bar{\varepsilon}$ & $93.1 \%$ & $15.9 \%$ & $7.6 \%$ & $4.7 \%$ & $1.7 \%$ \\
\hline \multirow{2}{*}{$\phi 50 \mathrm{~nm}$} & Position & $z^{+}=5$ & $z^{+}=10$ & $z^{+}=15$ & $z^{+}=20$ & $z^{+}=30$ \\
\cline { 2 - 7 } & $\bar{\varepsilon}$ & $45.8 \%$ & $4.2 \%$ & $-0.4 \%$ & $0.6 \%$ & $-0.7 \%$ \\
\hline
\end{tabular}

The measured values at $y=0$ for each value of $z^{+}$are plotted as the velocity profiles near the wall (Fig. 5). The measured data are obtained under $0.8,1.6$ and $2.4 \mathrm{kPa}$ pressures, respectively. The error bars are based on the standard deviations $\sigma$ in the ensemble average for each pressure. The velocity profiles are non-dimensionalized as $u^{+}=u / U_{\max }$, where $U_{\max }$ is the theoretical maximum velocity of flow in the channel. From Fig. 5a, for the experiments with $\$ 200 \mathrm{~nm}$ particles, at $z^{+}=7.5$, the measured velocity data are consistent with the theoretical no-slip values. But the measured velocity data obviously deviate from the theoretical no-slip values when $z^{+} \leqslant 5$, and the error is greater nearer the wall. The same tendency for $\phi 50 \mathrm{~nm}$ particles is shown in Fig. $5 \mathrm{~b}$, the deviation between measured data and theoretical values become obvious when $z^{+} \leqslant 10$. However, we notice that the deviation with $\phi 50 \mathrm{~nm}$ particles is much smaller than 
those with $\phi 200 \mathrm{~nm}$ particles. Quantitatively, with $\phi 50 \mathrm{~nm}$ tracer particles instead of $\phi 200 \mathrm{~nm}$ particles, the value of average relative deviation $\bar{\varepsilon}$ at the same position $z=250 \mathrm{~nm}$
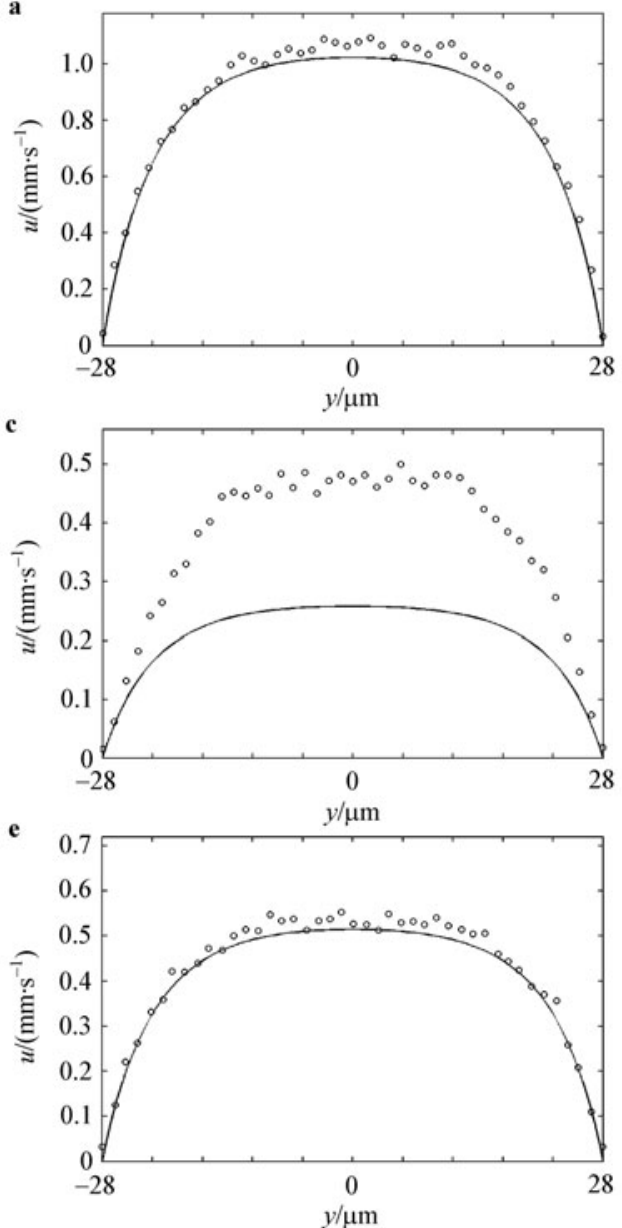

decreases from $93.1 \%$ to $45.8 \%$ (Table 1). It is clear that smaller particles size decreased measurement error.
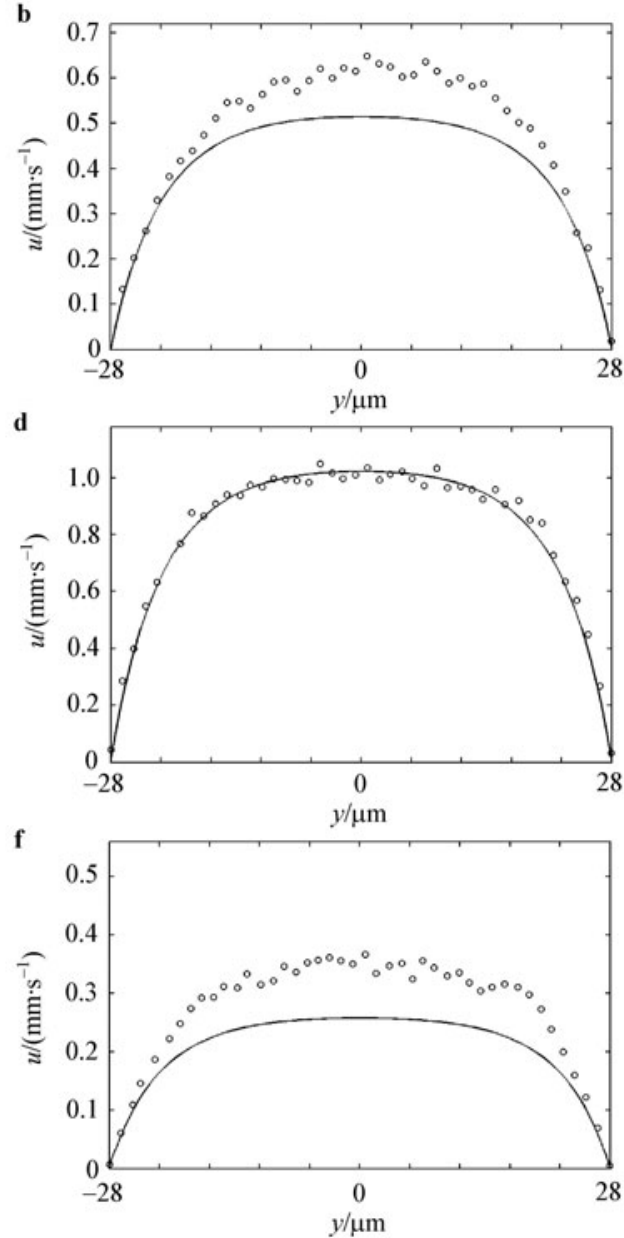

Fig. 4 The measured velocity data (circles) at three horizontal planes using $\phi 200 \mathrm{~nm}(\mathbf{a}-\mathbf{c})$ and $\phi 50 \mathrm{~nm}$ particles (d-f), compared with the theoretical no-slip profiles (Eq. (2), solid lines). The driven pressure was $2.4 \mathrm{kPa}$. a $z=1.0 \mu \mathrm{m}\left(z^{+}=5\right.$ ), $\phi 200 \mathrm{~nm} ; \mathbf{b} z=0.5 \mu \mathrm{m}\left(z^{+}=2.5\right)$, $\phi 200 \mathrm{~nm} ; \mathbf{c} z=0.25 \mu \mathrm{m}\left(z^{+}=1.25\right), \phi 200 \mathrm{~nm} ; \mathbf{d} z=1.0 \mu \mathrm{m}\left(z^{+}=20\right), \phi 50 \mathrm{~nm} ; \mathbf{e} z=0.5 \mu \mathrm{m}\left(z^{+}=10\right), \phi 50 \mathrm{~nm} ; \mathbf{f} z=0.25 \mu \mathrm{m}\left(z^{+}=5\right)$, $\phi 50 \mathrm{~nm}$
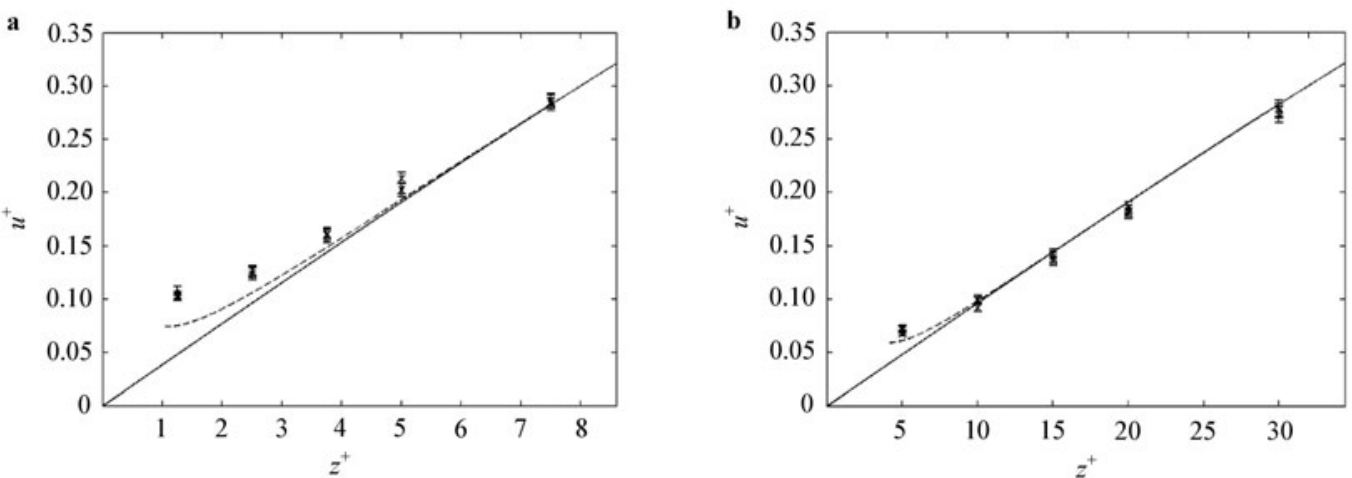

Fig. 5 A comparison of the dimensionless measured data (crosses with error bars), the theoretical velocity profile (solid line, Eq. (2)) and the predicted velocity (dash line, Eq. (8)) in the $x-o-z$ plane using $\mathbf{a} \phi 200 \mathrm{~nm}$ particles and $\mathbf{b} \phi 50 \mathrm{~nm}$ particles. The horizontal scale is dimensionless position $z^{+}\left(z^{+}=z / 2 r\right)$, and the vertical scale is $u^{+}\left(u^{+}=u / U_{\max }\right)$. The measured data were obtained under driven pressures of $0.8,1.6$ and $2.4 \mathrm{kPa}$, respectively 
In the present experiments, the Reynolds number are $R e=0.04-0.13$ and the influences of corner flow and secondary flow are omitted for such a low $R e$ [20]. The velocity measurements were repeated three times with different microchannels for both $\phi 50 \mathrm{~nm}$ and $\phi 200 \mathrm{~nm}$ particles, and the results were highly reproducible.

\subsection{The particle concentration distribution}

During the experiments, we also observed that the particle concentrations are not uniform in the near wall region. After filtering the out-of-focus particles in the images using a

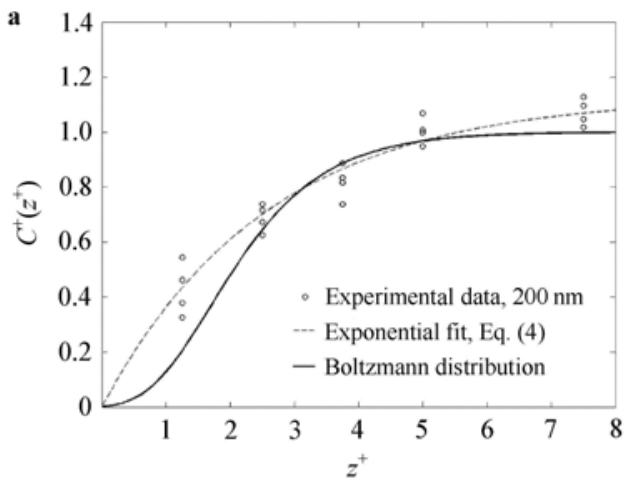

threshold value (Sect. 2.3), the particle concentration distribution could be obtained by counting the number of particles at each $z$ location. Approximately 3000 particles were counted from a series of 250 images at each plane. Figure 6 shows the non-uniform measured concentration distribution $c^{+}\left(c^{+}=c(z) / c_{0}, c_{0}\right.$ is the concentration in the main flow region far from the wall) along $z^{+}\left(z^{+}=z / 2 r\right)$. For $\phi 200 \mathrm{~nm}$ particles (Fig. 6a, circles), the particle concentration increases from $z^{+}=1.25-5$. For $\phi 50 \mathrm{~nm}$ particles (Fig. 6b, triangles), the particle concentration is low at $z^{+}=5$, and approximately constant when $z^{+} \geqslant 10$.

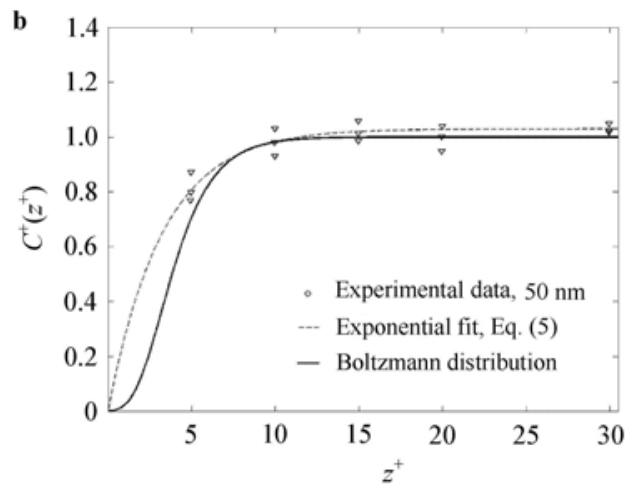

Fig. 6 The measured particle concentration distribution $c^{+}\left(z^{+}\right)$of a $\phi 200 \mathrm{~nm}$ particles and $\mathbf{b} \phi 50 \mathrm{~nm}$ particles, compared with the experimental fitting curves (dashed curves, Eqs. (4) and (5)) and Boltzmann distribution (solid curves, from Eqs. (6) and (7)) along the vertical $z^{+}$direction. The wall shear rates $\dot{\gamma}_{\mathrm{w}}$ are approximately $1200 \mathrm{~s}^{-1}$ (driven pressure $1.6 \mathrm{kPa}$ )

A dimensionless exponential particle concentration distribution in the wall depletion layer is given by Hartman Kok [14]. We fitted the experimental data with a dimensionless form of

$c^{+}\left(z^{+}\right)=\frac{n\left(z^{+}\right)}{n_{0}}=a_{1}\left(\mathrm{e}^{a_{2} z^{+}}-1\right)$,

where $n\left(z^{+}\right)$is the particle number count at position $z^{+}$, representing the concentration at position $z^{+}, n_{0}$ represents the uniform concentration in the main flow region, $a_{1}$ and $a_{2}$ are constants determined by fitting. For $\phi 200 \mathrm{~nm}$ particles, $n_{0}$ is the number count at $z^{+}=7.5$ from the image series $\left(n_{0} \approx 3700\right)$, and the fitting curve with $95 \%$ confidence is (dashed curve in Fig. 6a)

$c^{+}\left(z^{+}\right)=-1.13\left(\mathrm{e}^{-0.3874 z^{+}}-1\right)$.

For $\phi 50 \mathrm{~nm}$ particles, $n_{0}$ is the average number from $z^{+}=10$ to 30 . So the fitting result (dashed curve in Fig. 6b) is

$c^{+}\left(z^{+}\right)=-1.03\left(\mathrm{e}^{-0.3071 z^{+}}-1\right)$.

Furthermore, by introducing the electrostatic force, the hydrodynamic effect on the particles and the diffusion effects into the Nernst-Planck equation, Boltzmann concentration distribution can be solved to describe the bias particle concentration profile. The Boltzmann distribution can be expressed as

$c^{+}\left(z^{+}\right)=\exp \left(-\frac{q_{\mathrm{p}} \Psi\left(z^{+}\right)}{k_{\mathrm{B}} T}\right)$, where $q_{\mathrm{p}}$ is the particle surface charge, $k_{\mathrm{B}}$ is Boltzmann constant, $T$ is temperature, $\Psi$ is the electric potential created by wall [21]

$\Psi\left(z^{+}\right)=\frac{4 k_{\mathrm{B}} T}{q} \tanh ^{-1}\left(\tanh \left(\frac{q \zeta}{k_{\mathrm{B}} T}\right) \exp \left(-z^{+} \frac{2 r}{\lambda_{D}}\right)\right)$,

where $\zeta$ is the zeta potential of the glass surface, $\lambda_{\mathrm{D}}$ is the Debye length. In Fig. 6, the solid curves are plotted to represent Boltzmann distribution. It is clear that the measured data of $\phi 50 \mathrm{~nm}$ particles are in good agreement with Boltzmann distribution, though the measured data of $\phi 200 \mathrm{~nm}$ particles show a notable deviation from Boltzmann distribution which is due to the fact that $\phi 200 \mathrm{~nm}$ particle is too big for the submicron near wall region. An analysis [16] in our previous study has also shown that, at small or moderate shear rate $\left(\dot{\gamma}_{\mathrm{w}}<5000 \mathrm{~s}^{-1}\right)$ Boltzmann distribution is a proper approximation to illustrate the nano-particles concentration distribution near wall.

Thus, in the next section, in order to conveniently introduce the concentration distribution into the velocity profile correction, only Boltzmann distribution (Eqs. (6) and (7)) will be used.

\subsection{Velocity profile correction}

Figures $5 \mathrm{a}$ and $5 \mathrm{~b}$ show that the measurement data are inconsistent with the expected theoretical no-slip velocity profile in the near wall region when $z^{+} \leqslant 5$ for $\phi 200 \mathrm{~nm}$ and $z^{+} \leqslant 10$ 
for $\phi 50 \mathrm{~nm}$ measurements. Furthermore, the particle concentration distributions appeared to be biased when $z^{+} \leqslant 5$, and become uniform when $z^{+}>5-10$. Here, we will analyze the reason of velocity deviation near wall.

As mentioned in Sect. 2.3, the "effective focus plane thickness" $\Delta z$ is defined and evaluated to be approximately $400 \mathrm{~nm}$. The measured velocity at focus plane $z=z_{0}$ in one horizontal plane $\bar{u}\left(z_{0}\right)$ is actually a spatial average over the effective focus plane thickness $\Delta z$. However, if the particle concentration is not uniform in this thickness, a deviation of the measured velocity will be introduced. A schematic diagram of near wall region in the microchannel is shown in Fig. 7, including the shear flow velocity distribution $u(z)$, the particle concentration distribution $c(z)$ and the effective focus plane thickness $\Delta z$.

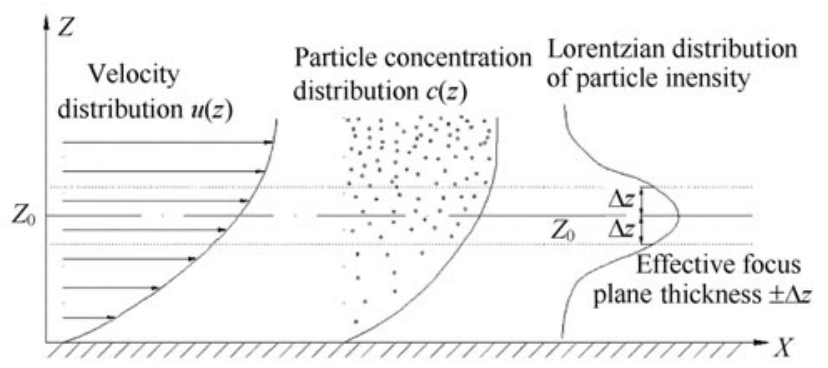

Fig. 7 A schematic image of the physical parameters involved in the near wall velocity measurements, including the shear flow velocity distribution $u(z)$, the particle concentration distribution $c(z)$ and the effective focus plane thickness $\Delta z$

To consider the influence of $c(z)$, a predicted average velocity for the measurement volume $\bar{u}^{\prime}\left(z_{0}\right)$ can be expressed as

$$
\bar{u}^{\prime}\left(z_{0}\right)=\frac{\int_{z_{0}-\Delta z / 2}^{z_{0}+\Delta z / 2} u(z) c(z) \mathrm{d} z}{\int_{z_{0}-\Delta z / 2}^{z_{0}+\Delta z / 2} c(z) \mathrm{d} z},
$$

where $u(z)$ and $c(z)$ are the distribution functions of the theo-

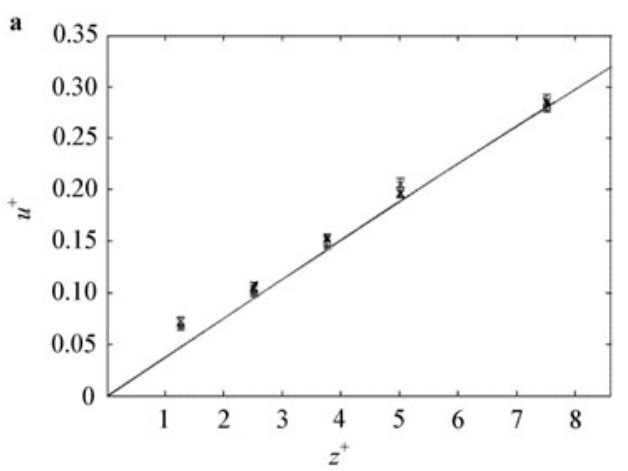

retical velocity and the Boltzmann distribution, respectively. The integral range is from $z_{0}-\Delta z / 2$ to $z_{0}+\Delta z / 2$, covering the whole effective focus plane thickness at the expected measurement position $z_{0}$. The theoretical no-slip velocity profile(Eq. (2)) is used to calculate $u(z)$, and the Boltzmann distribution (Eqs. (6) and (7)) is used to calculate $c(z)$ for $\phi 200 \mathrm{~nm}$ or $\phi 50 \mathrm{~nm}$ particles, respectively. A numerical integral method (Simpson quadrature) is used to calculate the predicted velocity profile $\bar{u}^{\prime}\left(z_{0}\right)$. Equation (8) shows that the deviation in $\bar{u}^{\prime}\left(z_{0}\right)$ depends strongly on the particle size (due to $c(z))$ and the effective focus plane thickness $\Delta z$, and clearly the corrected result of $\phi 50 \mathrm{~nm}$ particles is much better than that of $\phi 200 \mathrm{~nm}$ particles.

The predicted profile $\bar{u}^{\prime}\left(z_{0}\right)$ for $\phi 200 \mathrm{~nm}$ particles is added in Fig. 5a by a dashed line, and that for $\phi 50 \mathrm{~nm}$ particles is shown in Fig. 5b. The predicted velocity profile $\bar{u}^{\prime}\left(z_{0}\right)$ closely matches the measured velocities which deviates from the theoretical velocity profiles. The average relative deviation $\bar{\varepsilon}$ between the measured data and the predicted values $\bar{u}^{\prime}\left(z_{0}\right)$ for $\phi 50 \mathrm{~nm}$ particles at $z=0.25 \mu \mathrm{m}$ becomes $10.1 \%$ instead of $45.8 \%$. And $\bar{\varepsilon}$ for $\phi 200 \mathrm{~nm}$ is also reduced, which becomes $48.3 \%$ instead of $93.1 \%$. Thus we find that for microPTV measurements near a hydrophilic wall, biased velocity data will appear due to the non-uniform particle concentration in the effective focus plane thickness (Fig. 7).

The velocity deviation $\Delta u(z)$ due to the influences mentioned above can be calculated as $\Delta u(z)=\bar{u}^{\prime}(z)-u(z)$. Therefore, a corrected measured velocity $u_{\exp }^{*}(z)$ can be obtained as below to eliminate the influences

$u_{\exp }^{*}(z)=u_{\exp }(z)-\Delta u(z)$.

The corrected measured velocity data are presented in Figs. $8 \mathrm{a}$ and $8 \mathrm{~b}$, respectively. It is clear that after correction the deviation between the measured data and the no-slip theoretical velocity becomes much smaller. This result indicates that it should be very careful to make boundary slip conclusion by directly using microPIV/PTV data. Larger apparent measured velocities, even close to hydrophilic wall, are usually obtained due to the effects mentioned above.

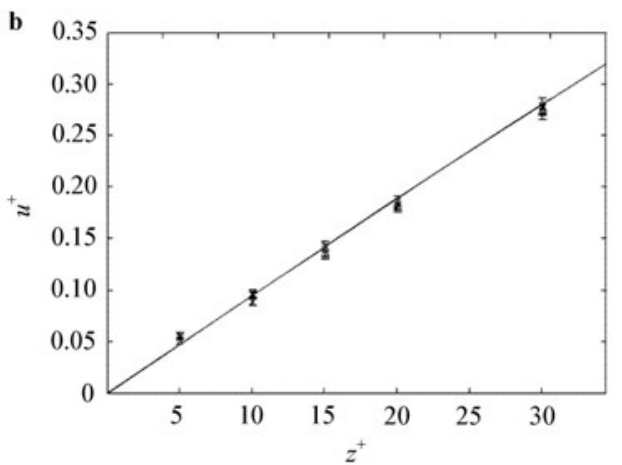

Fig. 8 A comparison of the corrected dimensionless measured velocity data (crosses with error bars, Eq. (9)) and the theoretical velocity profile (solid line, Eq. (2)) in the $x-o-z$ plane using a $\phi 200 \mathrm{~nm}$ particles and $\mathbf{b} \phi 50 \mathrm{~nm}$ particles. The correction is made based on Eq. (9) to eliminated the influence of the effective focus plane thickness combined with biased particle concentration distribution near wall. The measured data were obtained under driven pressures of $0.8,1.6$ and $2.4 \mathrm{kPa}$, respectively 


\subsection{Measured slip length}

Based on the measured velocity profile near wall, it is possible to get the information of boundary slip. The slip velocity can be evaluated by extrapolating the measured velocity profile to the wall with the shear rate as the slope, as illustrated in Fig. 1a. And then from Eq. (1), slip length $b$ can be calculated. Based on Fig. $8 \mathrm{~b}$ and the experimental data, we get $b$ being $(16 \pm 7) \mathrm{nm}$ ( $b$ is about $75 \mathrm{~nm}$ if no velocity correction was done, the diamonds in Fig. 9). Also, we retreat the data obtained by Joseph and Tabeling [5] with 100-200 nm particles, the slip length $b$ decreased approximately from $50 \mathrm{~nm}$ to $24 \mathrm{~nm}$ using the present correction method (the circles in Fig.9). The data from Huang and Breuer [15] were measured by total internal reflection velocimetry (TIRV) with evanescent wave, so it is beyond our discussion here. The slip length data in Fig. 9 show that by the present correction method, the deviation between the slip length measured by microPIV/PTV and that by SFA/AFM is decreased to $10 \mathrm{~nm}$ or so.

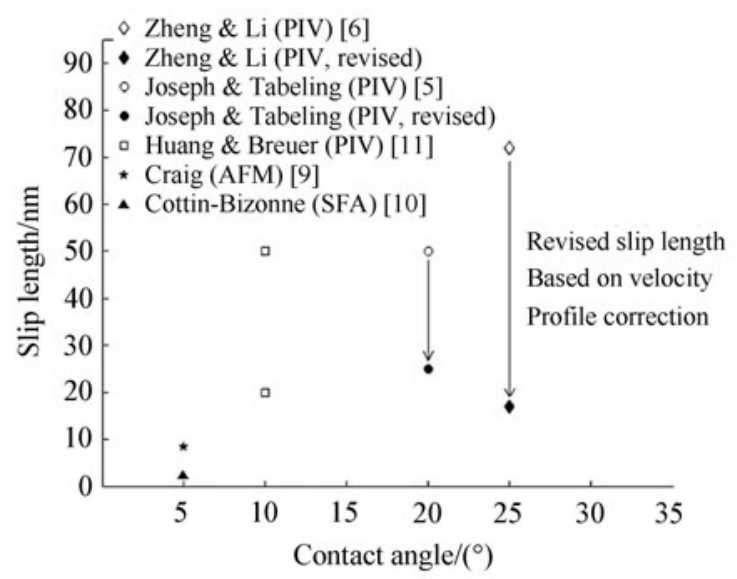

Fig. 9 A collection of slip length data measured by microPIV/PTV and SFA/AFM on hydrophilic walls. The measured slip length can be revised to $16 \mathrm{~nm}$ (the solid diamond) from the present measured data using $\phi 50 \mathrm{~nm}$ tracers, and about $24 \mathrm{~nm}$ (the solid circle) by the data from reference [5]. After correction, the deviation between the slip length measured by microPIV/PTV and that by SFA/AFM is decreased to $10 \mathrm{~nm}$ or so

However, the slip length is still $59 \mathrm{~nm}$ for $\phi 200 \mathrm{~nm}$ particles even after correcting the measured velocity profile. The explanation is that $\phi 200 \mathrm{~nm}$ particle is still too big for measuring velocity in the near wall region which is less than $500 \mathrm{~nm}$ measuring from the wall. Both the measured velocity profiles (Figs. 5 and 8 ) and the concentration distribution (Fig. 6) indicate that smaller particles lead to a better result and the particle size influence is important.

\section{Conclusions}

In this paper, detailed velocity measurements were carried out near a hydrophilic glass wall in a microchannel by a
microPTV method with $\phi 200 \mathrm{~nm}$ and $\phi 50 \mathrm{~nm}$ tracer particles. The near wall region was explored from $z=0.25 \mu \mathrm{m}$ to $z=1.5 \mu \mathrm{m}$ for steady laminar flow near a stationary smooth hydrophilic wall. The main results can be concluded as:

(1) The measured velocity data using $\phi 200 \mathrm{~nm}$ particles were significantly larger than the theoretical no-slip velocity at $z \leqslant 1.0 \mu \mathrm{m}\left(z^{+} \leqslant 5\right)$, the average relative deviation $\bar{\varepsilon}$ was $93.1 \%$ at $z=0.25 \mu \mathrm{m}\left(z^{+}=1.25\right)$. But for $\phi 50 \mathrm{~nm}$ particles, at the same vertical position, $z=0.25 \mu \mathrm{m}$ $\left(z^{+}=5\right), \bar{\varepsilon}$ decrease to $45.8 \%$. Thus smaller tracer particles can effectively reduce velocity deviations.

(2) For both $\phi 50 \mathrm{~nm}$ and $\phi 200 \mathrm{~nm}$ particles, the particle concentration distributions were uniform when $z^{+} \geqslant 10$, and it become obviously biased when $z^{+} \leqslant 5$. However, the concentration profile of $\phi 50 \mathrm{~nm}$ particles is more consistent with Boltzmann distribution than that of $\phi 200 \mathrm{~nm}$ particles. The deviations of near wall velocities measured by microPIV/PTV are strongly influenced by the biased particle concentration distributions. Based on the Boltzmann's exponential particle concentration distribution and the effective focus plane thickness, a predicted velocity profile is proposed. The relative deviation between the measured data and the predicted values $\bar{\varepsilon}$ at $z=0.25 \mu \mathrm{m}\left(z^{+}=5\right)$ can be reduced from $45.8 \%$ to $10.1 \%$ for $\phi 50 \mathrm{~nm}$ particles.

(3) By extrapolating the corrected measured velocity to the wall with measured shear rate as the slope, we found that the slip length (using $\phi 50 \mathrm{~nm}$ particles) decreased from $75 \mathrm{~nm}$ to $16 \mathrm{~nm}$, which approaches the slip length data obtained by other experimental method (SFA/AFM).

These results strongly suggest that the particle size and an exponential particle concentration distribution can significantly affect the near wall velocities measured by microPIV/PTV. Thus, the non-linear particle distribution in the shear flow should be considered in the interpretation of measured velocity and slip length in micrPIV/PTV measurement. A velocity correction method to eliminate the influences is also proposed. This result is very important for further practice of near wall velocity measurement such as nanoPIV, and a precise measurement of velocity profile close to wall will be also a foundation to study some other interfacial phenomena in micro/nanofluides, like the electrokinetic flow in the electric double layer.

Acknowledgement The authors gratefully acknowledge the discussions with Dr. C. Pipe and Dr. C. Cottin-Bizonne.

\section{References}

1 Navier, C.L.M.H.: Mémoire sur les lois du mouvement des fluids Mem. Acad. Sci. Inst. Fr. 6, 389 (1823)

2 Santiago, J.G., Wereley, S.T., Meinhart, C.D., et al.: A particle image velocimetry system for microfluidics. Exp Fluids 25, 316-319 (1998)

3 Meinhart, C.D., Wereley, S.T., Santiago, J.G.: PIV measurements of a microchannel flow. Exp. Fluids 25, 414-419 (1999) 
4 Tretheway, D.C, Meinhart, C.D.: Apparent fluid slip at hydrophobic microchannel wall. Phys. Fluids 14, L9-L11 (2002)

5 Joseph, P., Tabeling, P.: Direct measurement of the apparent slip length. Phys. Rev. E 71, 035303 (2005)

6 Zheng, X., Silber-Li, Z.H.: Measurement of velocity profiles in a rectangular microchannel with aspect ratio $\alpha=0.35$. Exp. Fluids 44, 951-959 (2008)

7 Lauga, E., Brenner, M.P., Stone, H.A.: Microfluidics: The noslip boundary condition. In: Handbook of Experimental Fluid Dynamics, Springer, New York (2005)

8 Neto, C., Evans, D.R., Bonaccurso, E., et al.: Boundary slip in Newtonian liquids: A review of experimental studies. Rep. Prog. Phys. 68, 2859-2897 (2005)

9 Craig, V.S.J., Neto, C., Williams, D.R.M.: Shear-dependent boundary slip in an aqueous Newtonian liquid. Physical Review Letters 87, 054504 (2001)

10 Cottin-Bizonne, C., Cross, B., Steinberger, A., et al.: Boundary slip on smooth hydrophobic surfaces: Intrinsic effects and possible artifacts. Physical Review Letter 94, 056102 (2005)

11 Huang, P., Guasto, J.F., Breuer, K.: Direct measurement of slip velocities using three-dimensional total internal reflection velocimetry. J. Fluid Mech. 566, 447-464 (2006)

12 Olsen, M.G., Adrian, R.J.: Out-of-focus effects on particle image visibility and correlation in microscopic particle image velocimetry. Exp. Fluids 29, S166-S174 (2000)
13 Lauga, E.: Apparent slip due to the motion of suspended particles in flows of electrolyte solutions. Langmuir 20, 8924-8930 (2004)

14 Hartman, Kok P.J.A., Kazarian, S.G., Briscoe, B.J., et al.: Effects of particle size on near-wall depletion in mono-dispersed colloidal suspensions. J. Colloid and Interface Science 280, 511-517 (2004)

15 Goldman, A.J., Cox, R.G., Brenner, H.: Slow viscous motion of a sphere parallel to a plane wall-II: Couette flow. Chem. Engng. Sci. 22, 653-660 (1967)

16 Zheng, X., Silber-Li, Z.H.: The influence of Saffman lift force on nanoparticle concentration distribution near a wall. Applied Physics Letters 95, 124105 (2009)

17 Inoue, H.S.: Video Microscopy. (2nd edn.). Plenum Press, Oxford (1997)

18 Meinhart, C.D., Zhang, H.: The flow structure inside a microfabricated inkjet prinhead. J. Mems. 9, 67-75 (2000)

19 White, F.: Viscous Fluid Flow. McGraw-Hill, Inc. New York, 123, (1974)

20 Schlichting, H.: Boundary Layer Theory. (7th edn.). Springer, New York, 613-614 (1979)

21 Bouzigues, C.I., Tabeling, P., Bocquet, L.: Nanofluidics in the debye layer at hydrophilic and hydrophobic surfaces. Phys. Rev. Letter 101, 114503 (2008) 